\title{
Experimental Modeling of Transition Layer at Phase Boundary in Composites
}

\author{
Vladimir Alexeevich Smirnov, Evgeniy Valerjevich Korolev, Alexandr Viktorovich Evstigneev \\ "Nanomaterials and Nanotechnology" Research and Educational Center, Moscow State University of Civil Engineering, \\ Moscow, Russian Federation \\ e-mail: smirnov@nocnt.ru
}

\begin{abstract}
Properties of composite materials with different matrices and disperse phases are greatly affected by phenomena occurring at the phase boundary with largest area. This is a boundary between matrix and fine filler. Numerous processes take place during preparation, homogenization and curing of the thermoset composites. While the achieved overall result is strengthening, the nature of mentioned processes is in many cases still unknown. In the present work we have carried out model study of transition layer which can be formed at the phase boundary during production of nanomodified epoxy composite. Raman spectroscopy is used for investigation of structure and composition. It is shown that silicone precursor is chemically inert to the matrix material. Application of such precursor leads to formation of low modulus transition layer between matrix and filler, thus promoting relaxation of internal stresses during curing process.
\end{abstract}

Keywords-thermoset composites, phase boundary, nanomodification

\section{INTRODUCTION}

Construction materials with unique combinations of operational properties can be obtained if we use thermosetting polymers as matrix materials. Usage of such polymers opens up possibility to achieve high value of elastic modulus (in case of continuous reinforcement fibers, one application is non-metallic reinforcement for cement concrete), high values of compressive and tensile strength, relatively high heat and fire resistance, high water, frost and chemical resistance $[1,2]$.

This is especially true for the construction composites with epoxy matrices. Numerous opportunities for optimization of operating characteristics of mentioned composites serve as foundations of the development of fundamentally new structures for civil and industrial construction.

At the same time, despite the fact that epoxy resins are manufactured on a permanent basis by various chemical industry enterprises, the costs of epoxy oligomers, intermediate products and epoxy adducts are relatively high. For this reason it can not be expected that polymer concrete with epoxy matrix will compete with traditional Portland cement concrete in most areas of construction. Usage of epoxy polymer composites is beneficial only in specific areas of construction. In particular, primary application of epoxy composites in construction is the creation of multifunctional wall coatings and floor coverings. High cost of epoxy oligomer dictates necessity to improve the values of operational properties in such a way that designed material will be cost-effective.

During the development of nanotechnology of material science, attention is focused on the certain method which allows improvement of operational properties. This method is called nanomodification, and in most cases it consists in creation of the nanoscale transitional layer on the interface boundaries between matrix and fine filler [2, 3]. Similar methods of directional control of structure formation were known earlier. However, till present, the mechanisms of the enhancement of macroscopic properties caused by the mentioned transition layer are subjects of debate (there are at least two distinct theories, namely theory of adhesive bond and theory of deformable layer). The situation is further complicated due to many types of epoxy oligomers, crosslinking agents and precursors of nanomodifiers.

Opening of the epoxide cycle in glycidyl functional group of epoxy oligomer may be initiated by various factors. As a consequence, there are several curing mechanisms of epoxy resins. Most important mechanism is the addition polymerization mechanism, which is (unlike the condensation) not accompanied by the detachment of extra substances (in this respect it is similar to the polymerization of most thermoplastics). Under normal conditions this curing mechanism of epoxy oligomers is realized for broad class of curing agents - aliphatic amines. Because of this, aliphatic amines are widely used in the production of epoxy composites. However, considering the production technology, many aliphatic amines (triethylenetriamine, polyethylenepolyamine etc.) are not without drawbacks. Due to significant difference in the mass fractions of the oligomer and hardener, high accuracy of dosage is required; due to substantial exothermic effect of the crosslinking, time boundaries of the production cycle are contracted and mixing technology is complicated.

Apparently, using triethylenetriamine and other pure amines as crosslinking agents is exclusively of academic interest and for laboratory studies. Polyethylenepolyamine, curing agent was widely accepted before, is a mixture of technical amines and different byproducts. Such curing agent was once significantly cheaper than pure aliphatic amines. At present, taking into account its numerous drawbacks, usage of polyethylenepolyamine can not considered as a reasonable choice.

Currently, whole series of products is positioned as a replacement for pure amine crosslinking agents. One of such products is OP-921 hardener. The compositions of the 
crosslinking agents are not disclosed, only technical specifications are available.

High-molecular silicone compounds with siloxane bond in the main chain are often used as precursors for nanomodification of epoxy composites. Typically, such compounds are synthesized by hydrolytic condensation of chlorosilanes, followed by polymerization. In the industry these organosilicon oligomers and polymers are used in form of solutions in aromatic solvents: toluene, xylene and others. Mass fraction of silicone polymer in the solution ranges from 30 to $50 \%$.

In particular, in Russian Federation there are several technical documents for regulating the compositions of silicone varnishes. KO-921 varnish (RU GOST 16508-70) can successfully be used for development of nanomodified epoxy composites [2, 4].

Nanomodification technology includes several steps:

- Design of composition, estimation of total area of phase boundary between fine filler and matrix material.

- Preparation of diluted solution of silicone compound in pure aromatic solvent;

- Processing of the fine filler with a solution of silicon compound.

- Drying of the fine filler.

- Combination processed fine filler, epoxy oligomer, crosslinking agent.

- Homogenization of the composition.

- Curing at optimal thermal conditions.

Improvement of operational performance properties of nanomodified epoxy composites is by now reliably established. At the same time, experimental study of the composition and structure of the resulting nanoscale boundary layer meets a number of objective difficulties.

\section{EXPERIMENTAL DESIGN}

To obtain the required information about boundary layer in nanomodified composites we have desing and implement "model" experiment. In this experiment we have studied the effect of macroscopic amounts of silicone modifier on the properties of the "oligomer-hardener" system. The feasibility of such study is determined by the basic provisions of the system analysis: to perform a complete decomposition of complex systems into components [5].

The epoxy compositions are prepared with bisphenolbased epoxy oligomer DER-330 and OP-921 curing agent. Silicone varnish $\mathrm{KO}-85$ is used as a precursor during nanomodification process. Oligomer DER-330 is produced by Dow Chemical company; in the industry, ASTM D-1652, ASTM D-1209, ASTM D-445, ASTM D-1726, ASTM E203, ASTM D-4052 are used for determination of properties, and residual epichlorohydrin is determined by the method disclosed in DowM 101321. Under normal conditions viscosity of the DER-330 oligomer is similar to viscosity ED-20 epoxy resin; ratio of epoxide groups is similar to one for ED-22 resin. While both ED-20 and ED-22 epoxy resins are widely used in construction, DER-330 is characterized by an order of magnitude smaller amount of the active chlorine content; there are almost no impurities. DER-330 is also characterized by small width of the molecular weight distribution. Unlike other aliphatic amine hardeners, OP-921 crosslinking agent is designed for using in mass ratio to oligomer which is close to $1: 1$. This simplifies the preparation technology of the composition. Other positive properties of OP-921 hardener are reduced exothermic effect of reaction and possibility (for an appropriate choice of the oligomer) to produce coatings with improved decorative properties. Preparation of test samples of epoxy resins includes dosage, homogenization and deposition on the substrate. Curing is performed under normal conditions, duration of curing is $24 \mathrm{~h}$.

Raman spectroscopy is used as a primary method of analysis. This method comprises registration of the spectral modes (frequencies of Raman shifts and relative intensities of shifted modes; latter are expressed in arbitrary units) of the scattered monochromatic electromagnetic wave. Raman spectroscopy allows to relax the requirements for sample preparation and the total amount of samples, and also is highly sensitive to any change in the composition of organic [6] and inorganic [7, 8] reagents. All measurements are performed on the Senterra confocal spectrometer. The parameters of the optical path during registration of reference spectra of triethylenetriamine, crosslinking agent OP-921 and epoxy oligomer DER-330, as well as spectra of the compositions at the time of gelation point, are set as follows: excitation wavelength is $532 \mathrm{~nm}$, excitation power is $10 \mathrm{~mW}$, slit aperture is 50 by $1000 \mathrm{um}$, accumulation time is $60 \mathrm{~s}$ (three consecutive measurements of the same duration are carried out). During registration of the spectra of fully cured products the excitation power is reduced down to $2 \mathrm{~mW}$. Spectral resolution is in range $3.5-9 \mathrm{~cm}^{-1}$, depending on the spectral window. The Raman shifts are recorded for the spectral range $45-4450 \mathrm{~cm}^{-1}$.

The study of model systems is carried out in accordance with the experimental design specific for "compositionproperty" ternary systems. The key idea is quite obvious: irrespectively to the exact reasons and mechanisms of the strengthening, the displacement of the precursor from the matrix and selective absorption take place during preparation and curing of the epoxy composition with fine filler. Moreover, for the selected type of curing agent, mass ratio of oligomer to hardener is also influence structure of the composition. Because of the latter, mentioned ratio is also included as variable. Thus, the variables are:

1. Mass ratio of crosslinking agent to oligomer:

$$
\alpha=\frac{m_{h}}{m_{r}} .
$$

2. Mass ratio of the modifier to epoxy binder:

$$
\beta=\frac{m_{m}}{m_{r}+m_{h}}
$$


In Eq. (1) and (2) values $m_{h}, m_{\mathrm{r}}$ and $m_{m}$ are masses of crosslinking agent, epoxy oligomer and modifier (silicone resin without solvent), respectively.

The set of equations

$$
\left\{\begin{array}{l}
\alpha=\frac{m_{h}}{m_{r}} \\
\beta=\frac{m_{m}}{m_{r}+m_{h}} \\
m_{h}+m_{r}+m_{m}=M
\end{array}\right.
$$

Where $M$ is the mass of composition, can be solved for the unknown masses:

$$
\left\{\begin{array}{l}
m_{r}=\frac{M}{(1+\alpha)(1+\beta)} \\
m_{h}=\frac{\alpha M}{(1+\alpha)(1+\beta)} \\
m_{m}=\frac{\beta M}{1+\beta}
\end{array}\right.
$$

Base levels and intervals of variations are

$$
\alpha_{0}=0,6, \Delta \alpha=0,2, \beta_{0}=0,3, \Delta \beta=0,3 \text {. }
$$

These values are used for the calculation of masses in the points “1"-“7” (Fig. 1). The vertices of concentration triangle correspond to the limiting values of input variables. These are so-called "basis compounds". Edge "3"-“2" correspond to the maximal concentration of the crosslinking agent, while edge " 1 "-“3” correspond to maximal concentration of modifier.

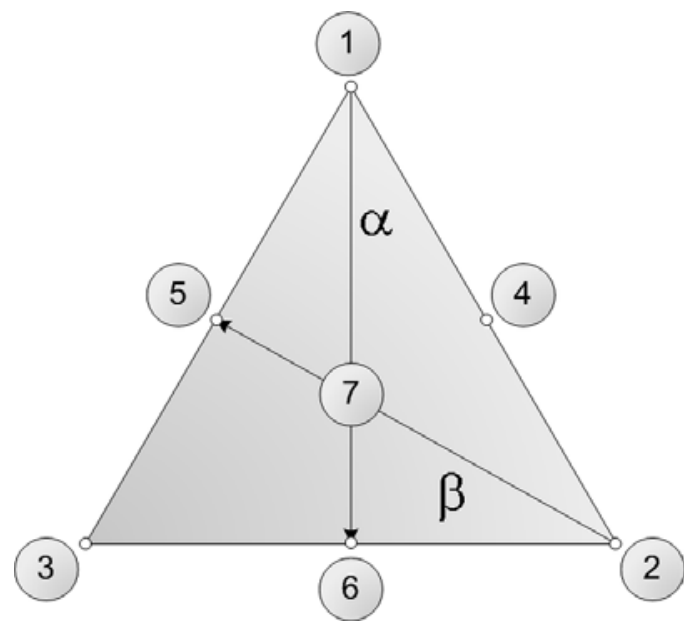

Figure 1. Concentration triangle: experimental design for model study of transition layer in epoxy composite

\section{RESULTS AND DISCUSSION}

The composition of the hardener OP-921 is not disclosed. Comparative analysis of the Raman spectra of triethylenetriamine, epoxy oligomer DER-330 and OP-921 crosslinking agent (Fig. 2) shows that OP-921 is probably and epoxyamine adduct. This is indicated by the coincidence of a well identifiable peaks at the frequencies 1460, 1550, 2900, 3060 and $3290 \mathrm{~cm}^{-1}$ (maximum at $1000 \mathrm{~cm}^{-1}$ may also indicate presence of aromatic additives in the OP-921). Raman spectra are recorded twice. The first series of measurements (Fig. 3-6) is carried out at the gelation point. The second series (Fig. 7-10) is performed for the fully cured composite.

Comparison of the Fig 1 and 3-6 shows that presence of the modifier has no significant impact on the structure of the curing product at the gelation point. In the Raman spectra of basis and central compositions there are just the same peaks as in the spectra on the reference oligomer and a hardener (Fig. 2). Formation of new chemical substances does not take place; silicone modifier is chemically inert to the matrix. The relative intensity of the peaks (if compared with (4) background caused by the formation of an irregular threedimensional spatial grid) begins to decline. This reduction is minimal for the basis composition "2" produced without modifier.

Upon completion of the curing process the overall character of Raman spectra transforms significantly. For the basis composition " 1 " with excessive amount of silicone modifier and epoxy oligomer (and, therefore, deficiency of the curing agent), Raman lines corresponding to a regular spatial grid of crosslinked thermoset can not be traced. Almost the same character of the Raman spectra holds for other compositions comprising a considerable amount of modifier. The irregularity of the spatial grid, with everything else being equal, corresponds to the low-modulus materials. Thus, it can be concluded that using the silicone precursors helps to create at the interface boundaries transition layer which reduces modulus of elasticity and leads to the relaxation of internal stresses of different origins. This conclusion is a very strong argument in favor of the theory of deformable layer.

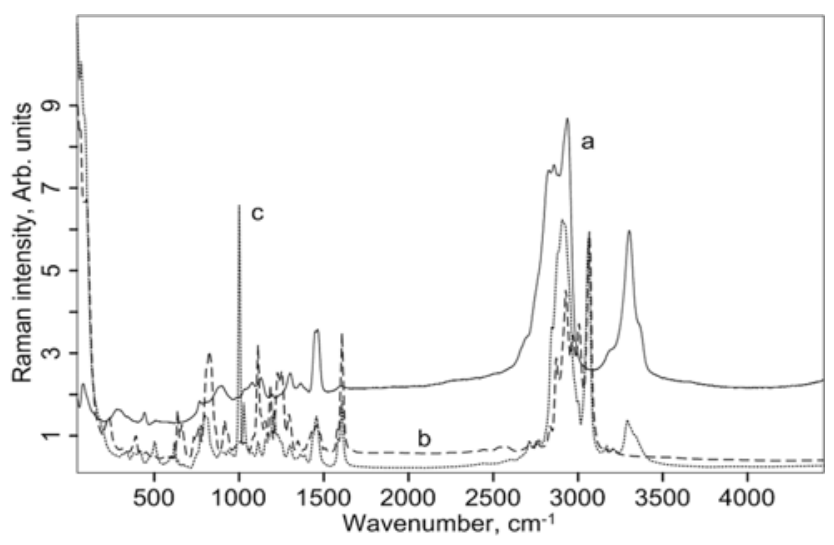

Figure 2. Raman spectra of triethylenetriamine (a), epoxy oligomer DER330 (b) and OP-921 crosslinking agent (c) 


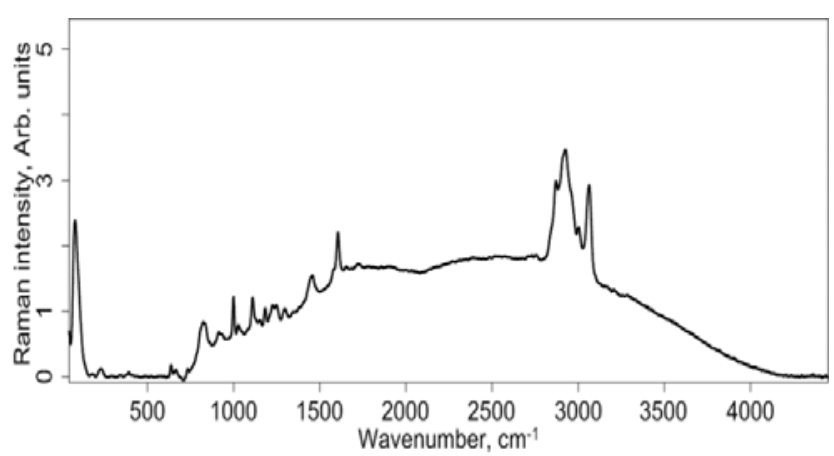

Figure 3. Raman spectrum of the basis composition "1", gelation point

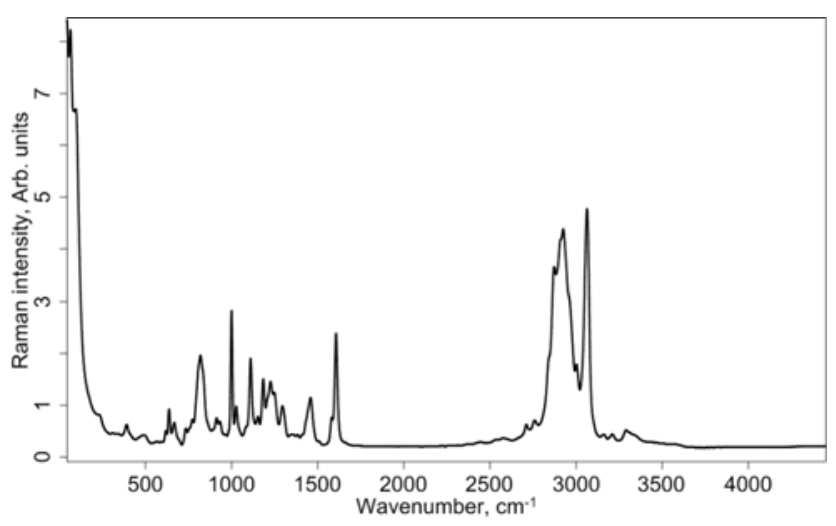

Figure 4. Raman spectrum of the basis composition "2", gelation point

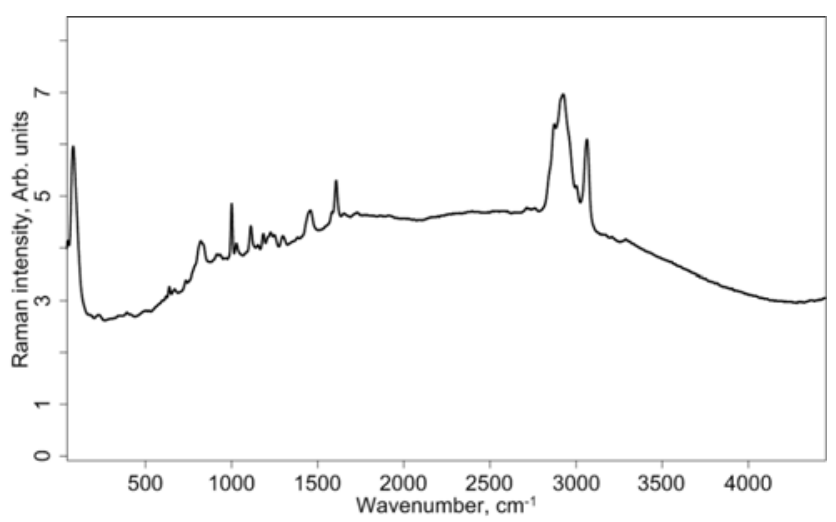

Figure 5. Raman spectrum of the basis composition “3”, gelation point

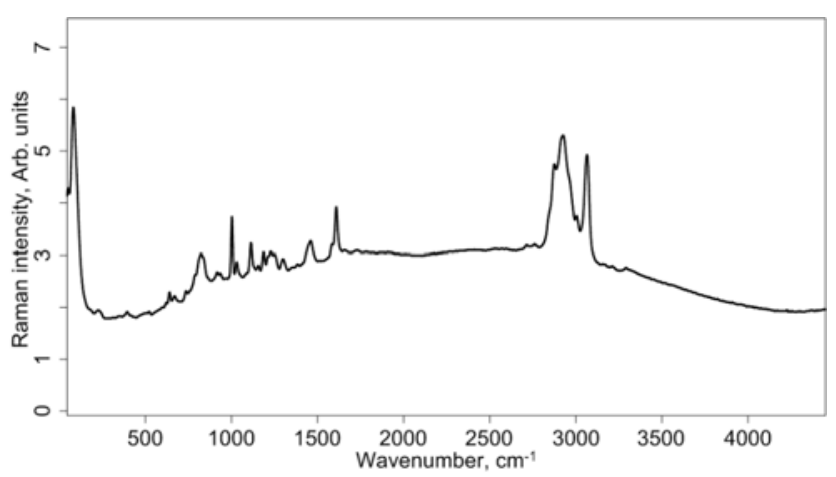

Figure 6. Raman spectrum of the central composition "7”, gelation point

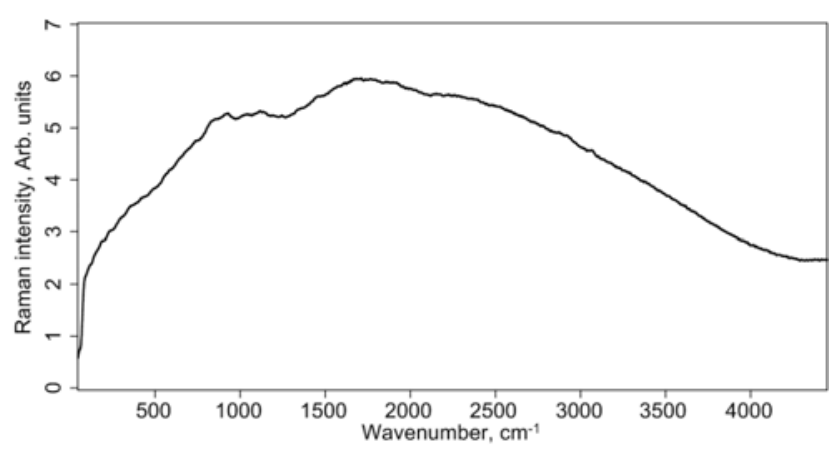

Figure 7. Raman spectrum of the cured basis composition " 1 ”

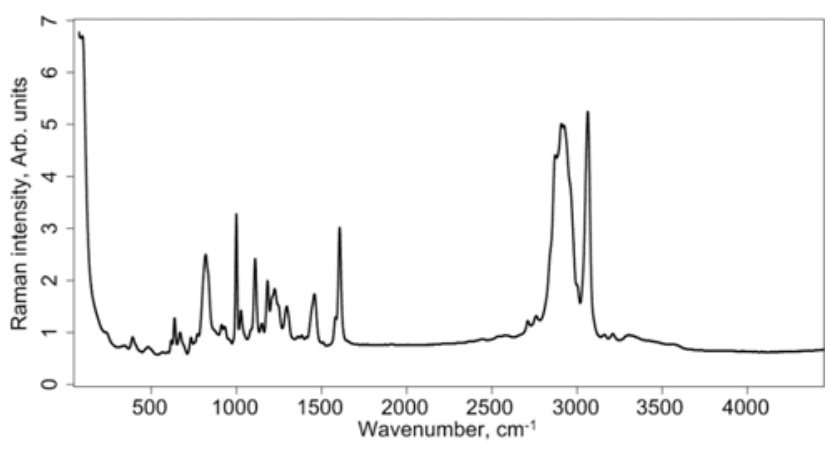

Figure 8. Raman spectrum of the cured basis composition "2"

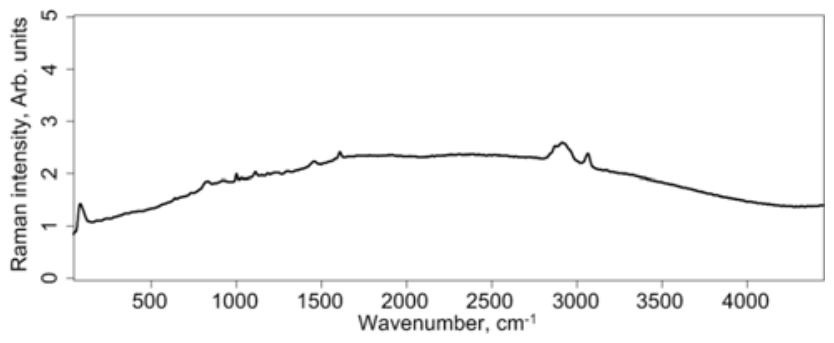

Figure 9. Raman spectrum of the cured basis composition " 3 ”

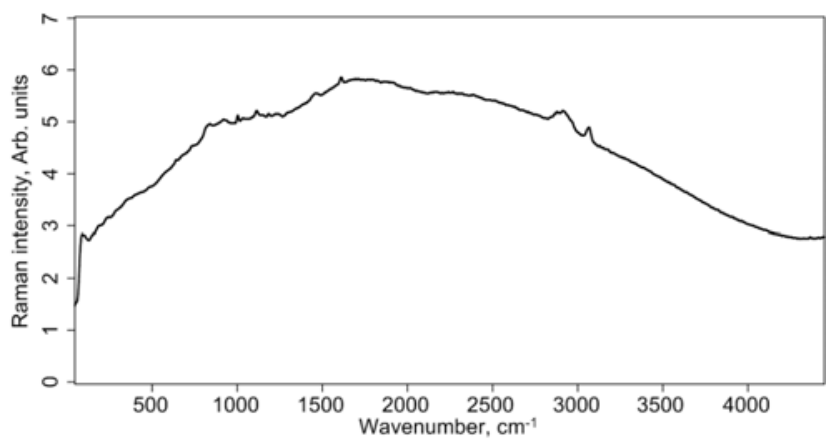

Figure 10. Raman spectrum of the cured central composition "7"

Quantitative assessment of the regularity of cross-linking can be done in various ways. In the simplest case it is sufficient to estimate the integrated intensity of the Raman spectrum. Such intensity is usually in positive correlation with "disorder" of the structure; spectra of most amorphous substances are without strongly expressed thin lines instead, spectrum is in form of intensive "background" with 
wide peaks. Due to the fact that the absolute value of integrated intensity depends on measurement setup and is expressed in relative units, it is expedient to normalize the value by means of division by highest value obtained for all seven investigated compositions. The regression model for the integrated intensity is:

$$
I=A+0,23 B-1,1 A B+0,46 A C+0,74 B C+2,14 A B C(6)
$$

Where $A, B$ and $C$ are fractions of the corresponding basis compositions.

The level set for (6) is shown on Fig. 11.

The character of level curves on Fig. 11 confirms the already mentioned circumstance: the spatial grid of most regularity is observed in case of the basis composition "2" without silicone modifier. At constant amount of modifier (edge "2"-“3" on Fig. 2) the introduction of an excess of crosslinking agent counteracts the effect caused by admixture of silicone modifier.

For some compositions (Fig. 7-10) the peaks are also observed at frequencies specific for spectrum of the crosslinking agent. This probably indicates that either hardener is exceeded stoichiometric amount, or curing process is not complete. The worst case is observed for the basis composition " 3 " which at the same time includes excess amounts of hardener and modifier.

The Raman lines which correspond to epoxy oligomer are expressed slightly. The cured matrix material is mostly amorphous for all basis compositions except “4”, “7” and " 6 ". It can be presumed that concentrations of modifier similar to ones observed in basis compositions " 4 ", "7" and "6" are realized in the transition layer by means of selective absorption of precursor during mixing and curing of nanomodified composite.

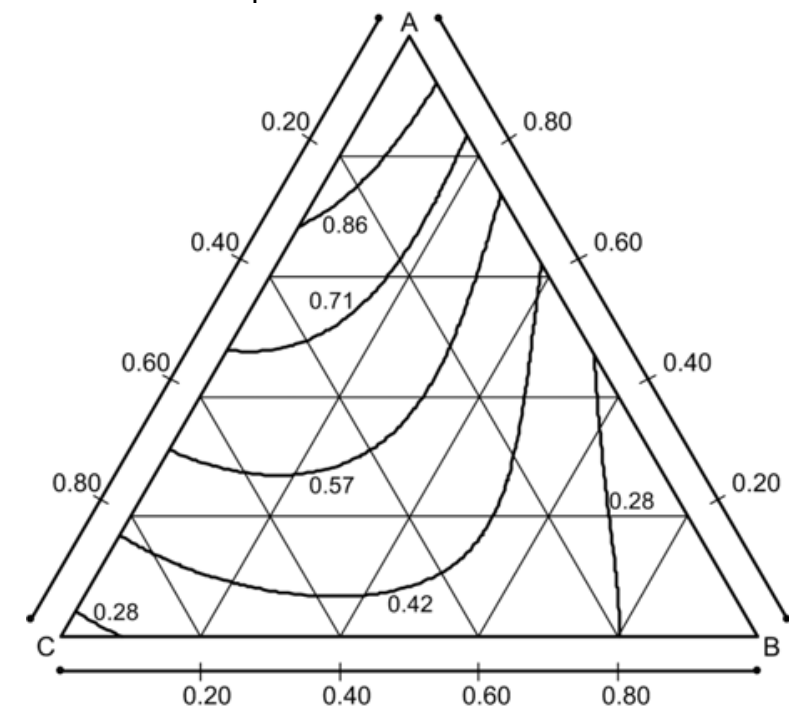

Figure 11. Level curves of relative integrated Raman intensity of the cured composition

\section{SUMMARY AND CONCLUSION}

Composites with a thermosetting matrix are often characterized by elevated values of operational properties flexural and compressive strength, resistance to aggressive environments, etc. At the same time, the cost of most thermosets (in particular, epoxy resins) is quite high. Because of this, the area of application of polymer composites in construction is limited. One such application is the creation of multifunctional coatings. The high cost of resin dictates the need to improve the operational properties to ensure economic efficiency. So far, the known way to improve the operational properties is to produce the nanoscale interfacial layer between fine filler and matrix in block. This way proved to be effective, but mechanism of the improvement is still uncertain. There exist at least two different theories - so-called "adhesion theory" and "theory of deformable layer".

In the present work we have used Raman spectroscopy for investigation of structure of cured epoxy resin with excessive amounts of silicone precursor which is usually used during nanomodification. It is shown that such precursor is chemically inert to the matrix material. Nevertheless, admixture of modifier reduces the regularity of the emerging three-dimensional spatial net of thermoset. After completion of the curing process the irregular spatial grid is still present; thus, the admixture of siloxane may lead to the formation of the transition layer with reduced modulus between matrix and filler. Such layer promotes relaxation of internal stresses during preparation of the composite. The overall effect of nanomodification consists in increased values of operational properties.

\section{ACKNOWLEDGMENT}

This work is supported by the Ministry of Science and Education of Russian Federation, Project \#2014/107, project title "Structure formation of sulfur composites: phenomenological and ab initio models”.

\section{REFERENCES}

[1] V.G. Khozin, Strengthening of the epoxy polymers. Kazan: PIC "Publishing House", 2004.

[2] V.A. Smirnov and E.V. Korolev, "Nanomodified epoxy composites," Nanotechnologies in Construction, 4, 2012, pp. 61-69.

[3] E.V. Korolev, V.A. Smirnov and A.I. Albakasov, "Nanomodified composites with thermoplastic matrix," Nanotechnologies in Construction, 5, 2012, pp. 81-87.

[4] RU Patent \#2488563, "Nanomodified Polymeric Composite.”

[5] Yu.M. Bazhenov, I.A. Garkina, A.M.Danilov and E.V Korolev, System analysis in Construction Material Science. Moscow: Moscow State University of Civil Engineering, 2012.

[6] K. Krishnan, "The Raman spectra of organic compounds," Proceedings of the Indian Academy of Sciences - Section A., vol. 53, 3, 1961, pp. 151-167, doi:10.1007/BF03045765

[7] E.V.Korolev, V.A. Smirnov and A.N. Zemlyakov, "Identification of the ASR products," News of the Moscow State University of Civil Engineering, 6, 2013, pp. 109-116.

[8] E.V. Korolev, S.V. Eskin and V.A. Smirnov, Raman spectroscopy during formation of silica gel," Nanotechnologies in Construction, 6, 2013,

pp.

$108-118$. 